\title{
Morphological Responses of Rice (Oryza sativa L.) to Organic and Inorganic Nutrients in Rice-Wheat Cropping System
}

\author{
Sanjay Kumar* and J. P. Srivastava \\ Department of Plant Physiology, Institute of Agricultural Sciences, Banaras Hindu University, \\ Varanasi, India-221005, India \\ *Corresponding author
}

\begin{tabular}{|l|}
\hline K e y w or d s \\
$\begin{array}{l}\text { Customized } \\
\text { fertilizer, Inorganic } \\
\text { nutrients, } \\
\text { Morphological }\end{array}$ \\
\hline Article Info \\
\hline $\begin{array}{l}\text { Accepted: } \\
\text { 08 January } 2020 \\
\text { Available Online: } \\
\text { 10 February } 2020\end{array}$ \\
\hline
\end{tabular}

\section{Introduction}

The rice (Oryza sativa L.) and wheat (Triticum aestivum L.) cropping system is found on 13.5 million hectares in South Asia and is one of the most important cropping patterns for food self-security in the region. This system is found in the fertile, hot semiarid to hot sub-humid regions of the
The present investigation deals with the effect of organic and inorganic sources of nutrient on morphological parameters of rice in rice-wheat cropping system. A field experiment was conducted at the experimental farm of the Institute of Agricultural Sciences, Banaras Hindu University, Varanasi, kharif and rabi season during 2014-15 and 2015-16. The experiment carried out in randomized block design with three replication and seven treatments viz. (Control; NPK 00:00:00)T1, (recommended dose of fertilizer: RDF; NPK 150:60:60) T2, (RDF + $\mathrm{S}: \mathrm{Zn}: \mathrm{B}$ 40:05:1.5) T3, (Customized fertilizer of Tata Chemical N:P:K:Zn:B: 11:32:13:0.9:0.24\%@375 kg ha ${ }^{-1}+179.34 \mathrm{~kg} \mathrm{ha}^{-1}$ urea in splits) T4, (75\% RDF $+25 \% \mathrm{~N}$ through sewage sludge) $\mathrm{T} 5,(75 \% \mathrm{RDF}+25 \% \mathrm{~N}$ through vermicompost) T6, (75\% RDF $+25 \% \mathrm{~N}$ through sesbania (GM)) T7. Results showed that the application of organic and inorganic sources of nutrients improved plant height, tillers hill ${ }^{-1}$, leaf number hill ${ }^{-1}$, leaf area $\mathrm{cm}^{2}$ hill $^{-1}$, leaf dry weight hill ${ }^{-1}$ and total plant dry weight hill ${ }^{-1}$. Improvements in treatments which were provided with inorganic nutrients, that too when RDF was supplemented with S, Zn and B (T3). Customized fertilizer of Tata Chemical (T4) was also effective in improving these parameters, but to a lesser magnitude than $\mathrm{T} 3$ in rice-wheat cropping system. 
tonnes of respectively. However, the productivity of both rice and wheat is low i.e. 2,424 and $3075 \mathrm{~kg} \mathrm{ha}{ }^{-1}$, respectively (Anonymous, 2015). Rice is mostly grown in lowlands under fully irrigated or rain-fed conditions. Organic materials particularly farmyard manure and green manures have traditionally been used by rice farmers in preindustrial age.

But with the present day high yielding cultivars, which have higher nutrient requirements, the use of inorganic fertilizers has increased considerably leading to decline in the use of organic materials (Hosain and Singh 2000). Both the crops grown in sequence require high quantity of nutrients to harvest their potential yields. The system overall is input intensive. These two crops remove more than $600 \mathrm{~kg}$ of NPK nutrients per ha annually when grown under good management conditions (Singha, 2003) With high yielding varieties, bumper crop of rice and wheat was harvested during the period of green revolution under sub-optimum levels of nitrogenous fertilizer alone.

Whatever, the reason behind this, but the consequences of continuous cropping with high yielding varieties and $\mathrm{N}$ fertilizer alone resulted in declining crop yields and soil fertility over years (Nambiar and Abrol, 1989). Use of organic and inorganic nutrients alone or with their integration (Bisht et al., 2002) has been found important for food security as well as soil sustainability. Vermicompost has been found five times richer in $\mathrm{N}$, seven times in $\mathrm{P}, 11$ times in $\mathrm{K}$, two times in $\mathrm{Mg}$ and $\mathrm{Ca}$, and seven times in Actinomycetes than in the ordinary soil (Mahajan and Gupta, 2009).

Sewage sludge is a good source of organic matter and contains good amounts of major and micro nutrients, which could be used as a supplement of major nutrients and fulfill the needs of micronutrients (Anonymous, 2013). Organic manures, particularly green manures and FYM, not only supply macronutrients but also meet the requirement of micronutrients, besides improving soil health. Boosting yield, reducing production cost and improving soil health are three inter-linked components of the sustainability triangle. Therefore, suitable combination of chemical fertilizers, organic manures and microbial cultures need to be developed for particular cropping system and soil (Bhasker, 2003).

Most soils of the rice-wheat cropping system in the Indo-Gangetic plains are deficient in $\mathrm{N}$ and deficiency of $\mathrm{K}$ is emerging in several areas (Alam et al., 2006). Among the $\mathrm{Zn}$ sources zinc sulphate $\left(\mathrm{ZnSO}_{4} \cdot 7 \mathrm{H}_{2} \mathrm{O}\right)$ is the most effective in making $\mathrm{Zn}$ readily available in soils. (Bodruzzaman et al., 2010) reported that the combined use of organic manure with inorganic fertilizers performed better than sole inorganic fertilizer to sustain the soil fertility and rice-wheat productivity. Therefore, keeping this in view, the present study was undertaken to investigate role of different fertilizers consisting of organic, inorganic and integrated sources of nutrient.

\section{Materials and Methods}

In present investigation, rice was grown at the experimental farm of the Institute of Agricultural Sciences, Banaras Hindu University, Varanasi, two seasons kharif and rabi during 2014-15 and 2015-16 and the results are average of these 2 years data. The experiment involved seven treatments in randomized block design with three replications. In This experiment 30 days old seedlings of rice variety HUR 105 were transplanted under following treatments: T1 (Control; NPK 00:00:00), T2 (recommended dose of fertilizer: RDF; NPK 150:60:60)], T3 (RDF + S:Zn:B: 40:05:1.5), T4 (Customized fertilizer of Tata Chemical having 
$\mathrm{N}: \mathrm{P}: \mathrm{K}: \mathrm{Zn}: \mathrm{B}: 11: 32 ; 13: 0.9: 0.24 \% @ 375 \mathrm{~kg}$ $\mathrm{ha}^{-1}+179.34 \mathrm{~kg} \mathrm{ha}^{-1}$ urea in splits), T5 (75\% $\mathrm{RDF}+25 \% \mathrm{~N}$ through sewage sludge), T6 $(75 \% \mathrm{RDF}+25 \% \mathrm{~N}$ through vermicompost), $\mathrm{T} 7$ (75\% RDF+ 25\% N through sesbania).

Observations on rice were recorded in three replications from three randomly selected plants (Hills). For each replication three plants were selected in each of the plot. Mean values of such three plants was considered as one replication in each treatment at a particular stage. All morphological observations in rice were made on per hill basis at 20,40,60, 80 days after transplanting and maturity stages viz. Plant height, tillers hill- ${ }^{1}$, Leaf number hill- ${ }^{1}$, Leaf area hill- ${ }^{1}$ and total dry weight of plant were as per standard procedure during 2014-15 and 2015-16 are follows:

\section{Plant height (cm)}

Height of the rice plants were measured from the base of the plant (soil surface) up to the tip of the growing plants with meter scale and expressed in centimeters. It was recorded at 20, 40, 60 and80, days after transplanting (DAT) and at maturity.

\section{Leaf number hill ${ }^{-1}$}

The number of green leaves per hill was counted separately at 20, 40, 60, 80 days after transplanting.

\section{Number of tillers hill-1}

Number of tillers hill ${ }^{-1}$ was counted at 20, 40, 60, 80 DAT and at maturity stages.

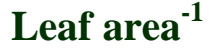

One rice hills from each replication were randomly harvested. Green leaves were separated immediately and area of total green leaves hill $^{-1}$ was determined by portable laser leaf area meter (CI-202, CID Bio Science, U.S.A.). It was expressed as $\mathrm{cm}^{2}$ hill $^{-1}$. Leaf area was measured from 20 DAT at an interval of 20 days till 80 days after transplanting.

\section{Dry weight (g) hill ${ }^{-1}$}

Plant samples (one hill) from each replication were harvested at an interval 20 days from 20 DAT till maturity and oven dried at $105^{\circ} \mathrm{C}$ for 15 min followed by at $65^{\circ} \mathrm{C}$ till constant weight. Plant dry weight was expressed as $\mathrm{g}$ hill $^{-1}$. At maturity leaves, stem and grain were separated and oven dried as explained above. Different plant parts were weighed with the help of electronic balance (Model; ADGR200) and their weights were expressed on hill ${ }^{-}$ ${ }^{1}$ basis.

Statistical analysis was done by method of analysis of variance for factorial randomized block design critical differences were calculated at 5\% level of significance in order to compare the treatment mean (Gomez and Gomez, 1984).

\section{Experimental results}

\section{Plant height (cm)}

Effect of different treatments on plant height of rice during 2014-15 is given in Table 1. Differences with respect to treatment (T), stages $(\mathrm{S})$, treatment $\times$ stage $(\mathrm{T} \times \mathrm{S})$ were significant. Plant height under all treatments increased steadily from transplanting to maturity stages.

At all stages it was the maximum in plants under T3 $(101.66 \mathrm{~cm})$ treatment, i.e., when plants received recommended doses of $\mathrm{N} P$ and $\mathrm{K}(\mathrm{RDF})$ and 40:05:1.50 kg ha ${ }^{-1}$ of $\mathrm{S}, \mathrm{Zn}$ and $\mathrm{B}$, respectively. At all stages the minimum plant height was evident in plants 
under T1 $(80.00 \mathrm{~cm})$ (control) and the maximum $(101.66 \mathrm{~cm})$ in plants under $\mathrm{T} 3$ treatment. Nevertheless, at maturity plant mean plant height under different treatments followed a trend as: $\mathrm{T} 3>\mathrm{T} 4>\mathrm{T} 2>\mathrm{T} 5>\mathrm{T} 6$
$>\mathrm{T} 7>\mathrm{T} 1$. During 2015-16 almost similar pattern was observed (Table 2). It was also evident that during 2015-16, under all treatments plants were relatively taller than during 2014-15.

Table.1 Plant height $(\mathrm{cm})$ in rice genotype HUR 105 at different stages of growth and treatments (2014-15)

\begin{tabular}{|c|c|c|c|c|c|c|c|}
\hline \multirow[t]{2}{*}{ S.No. } & \multirow[t]{2}{*}{ \# Treatment } & \multicolumn{6}{|c|}{ Plant height $(\mathrm{cm})$ at different days after transplanting } \\
\hline & & 20 & 40 & 60 & 80 & Maturity & Mean \\
\hline 1 & T1 & 39.00 & 51.00 & 63.50 & 73.33 & 80.00 & 61.37 \\
\hline 2 & T2 & 43.00 & 62.00 & 79.33 & 87.70 & 97.83 & 73.97 \\
\hline 3 & T3 & 46.00 & 66.66 & 83.33 & 99.00 & 101.66 & 79.33 \\
\hline 4 & T4 & 42.33 & 61.66 & 81.66 & 96.93 & 100.9 & 76.70 \\
\hline 5 & T5 & 43.33 & 57.33 & 77.66 & 90.66 & 96.33 & 73.07 \\
\hline 6 & T6 & 44.00 & 60.66 & 76.00 & 87.00 & 93.33 & 72.20 \\
\hline 7 & T7 & 42.83 & 57.00 & 75.33 & 88.40 & 93.16 & 71.35 \\
\hline \multicolumn{2}{|r|}{ Mean } & 42.93 & 59.48 & 76.69 & 89.00 & 94.75 & \\
\hline \multicolumn{2}{|c|}{ Particulars } & \multicolumn{3}{|c|}{ SEm \pm} & \multicolumn{3}{|c|}{$\mathrm{CD}(\mathrm{P} \leq \mathbf{0 . 0 5})$} \\
\hline \multicolumn{2}{|c|}{ Treatment } & \multicolumn{3}{|c|}{0.62} & \multicolumn{3}{|c|}{$1 . \overline{76}$} \\
\hline \multicolumn{2}{|r|}{ Stage } & \multicolumn{3}{|c|}{0.52} & \multicolumn{3}{|c|}{1.48} \\
\hline \multicolumn{2}{|c|}{ Treatment $\times$ Stage } & \multicolumn{3}{|c|}{1.39} & \multicolumn{3}{|c|}{3.93} \\
\hline \multicolumn{8}{|c|}{$\begin{array}{l}\text { \# (Control; NPK 00:00:00)T1, (recommended dose of fertilizer: RDF; NPK 150:60:60) T2, (RDF + S:Zn:B 40:05:1.5) T3 } \\
\text { (Customized fertilizer of Tata Chemical N:P:K:Zn:B: 11:32:13:0.9:0.24 \% @ } 375 \mathrm{~kg} \mathrm{ha}^{-1}+179.34 \mathrm{~kg} \mathrm{ha}^{-1} \text { urea in splits) T4, 75\% } \% \text { RDF + 25\% N through sewage sludge (T5), 75\% RDF + 25\% N through vermicompost (T6), 75\% RDF + 25\% N through sesbania } \\
\text { (GM) (T7). *Statistically not significant }\end{array}$} \\
\hline
\end{tabular}

Table.2 Plant height $(\mathrm{cm})$ in rice genotype HUR 105 at different stages of growth and treatments (2015-16)

\begin{tabular}{|c|c|c|c|c|c|c|c|}
\hline \multirow[t]{2}{*}{ S.No. } & \multirow[t]{2}{*}{ \# Treatment } & \multicolumn{6}{|c|}{ Plant height in $(\mathrm{cm})$ at different days after transplanting } \\
\hline & & 20 & 40 & 60 & 80 & Maturity & Mean \\
\hline 1 & T1 & 35.62 & 54.50 & 65.10 & 74.98 & 84.57 & 62.95 \\
\hline 2 & $\mathbf{T} 2$ & 43.67 & 67.37 & 83.30 & 90.57 & 100.50 & 77.08 \\
\hline 3 & T3 & 51.03 & 72.47 & 86.83 & 106.00 & 106.77 & 84.62 \\
\hline 4 & T4 & 50.37 & 70.67 & 85.53 & 101.80 & 104.67 & 82.61 \\
\hline 5 & T5 & 42.50 & 66.17 & 81.20 & 91.97 & 98.73 & 76.11 \\
\hline 6 & T6 & 41.33 & 65.97 & 79.07 & 91.10 & 98.73 & 75.24 \\
\hline 7 & \begin{tabular}{|l|}
$\mathbf{T 7}$ \\
\end{tabular} & 42.20 & 66.33 & 77.33 & 90.87 & 98.53 & 75.05 \\
\hline \multicolumn{2}{|c|}{ Mean } & 43.82 & 66.21 & 79.77 & 92.47 & 98.93 & \\
\hline \multicolumn{2}{|c|}{ Particulars } & \multicolumn{3}{|c|}{ SEm \pm} & \multicolumn{3}{|c|}{$\mathrm{CD}(\mathrm{P} \leq \mathbf{0 . 0 5})$} \\
\hline \multicolumn{2}{|c|}{ Treatment } & \multicolumn{3}{|c|}{0.68} & \multicolumn{3}{|c|}{1.92} \\
\hline \multicolumn{2}{|c|}{ Stage } & \multicolumn{3}{|c|}{0.57} & \multicolumn{3}{|c|}{1.62} \\
\hline \multicolumn{2}{|c|}{ Treatment $\times$ Stage } & \multicolumn{3}{|c|}{1.51} & \multicolumn{3}{|c|}{4.23} \\
\hline
\end{tabular}

\# (Control; NPK 00:00:00)T1, (recommended dose of fertilizer: RDF; NPK 150:60:60) T2, (RDF + S:Zn:B 40:05:1.5) T3, (Customized fertilizer of Tata Chemical N:P:K:Zn:B: 11:32:13:0.9:0.24\% @ $375 \mathrm{~kg} \mathrm{ha}^{-1}+179.34 \mathrm{~kg} \mathrm{ha}^{-1}$ urea in splits) T4, 75\% $\mathrm{RDF}+25 \% \mathrm{~N}$ through sewage sludge (T5), $75 \% \mathrm{RDF}+25 \% \mathrm{~N}$ through vermicompost (T6), $75 \% \mathrm{RDF}+25 \% \mathrm{~N}$ through sesbania (GM) (T7).* Statistically not significant 


\section{Tiller number hill ${ }^{-1}$}

During transplanting generally 2 rice plants hill $^{-1}$ were transplanted. Data regarding tiller hill $^{-1}$ during 2014-15 are given in Table 3.). Significant differences were recording between treatment, stage and treatment $x$ stage. Even after 20 days after transplanting (DAT) as compared to control (T1) tillers hill ${ }^{1}$ were significantly higher in plant under rest of the treatments; with the maximum in plants under T3 treatment i.e. 6 tiller hill ${ }^{-1}$. With advancement in stage tillers hill ${ }^{-1}$ increased steadily under all treatments.

At maturity stage mean tillers plant ${ }^{-1}$ were the maximum $\left(13.67 \mathrm{hill}^{-1}\right)$ in plants under T3 treatment and the minimum $\left(9.00 \mathrm{hill}^{-1}\right)$ in plans under $\mathrm{T} 1$ treatments. At maturity tillers hill $^{-1}$ under different varied as: $\mathrm{T} 3>\mathrm{T} 4>\mathrm{T} 2$ $>\mathrm{T} 7>\mathrm{T} 6>\mathrm{T} 5>\mathrm{T} 1$. Differences between T3 and $\mathrm{T} 4$ and $\mathrm{T} 2$ were rather marginal. Almost similar result were recorded during 2015-16, however, as compared to 2014-15, during
2015-16 tillers hill ${ }^{-1}$ in T1 decreased, while in plants under the other treatments it improved (Table 4).

\section{Leaf number hill ${ }^{-1}$}

Leaf number hill $^{-1}$ during 2015-16 showed significant differences with respect to treatment, stage and treatment $\times$ stage (Table 5). Differences were evident at 20 DAT stage with the maximum number of leaves in T3 and the minimum in $\mathrm{T} 1$ treatment. With advancement in stage, on an average leaf number hill ${ }^{-1}$ increased till 60 DAT and then declined. The maximum leaves hill $^{-1}$ at 80 DAT (51.00 hill-1) was observed in plant under T3 treatment and the minimum (22.00 hill $^{-1}$ ) in plants under T1 treatment. Under different treatments at 80 DAT leaves hill $^{-1}$ varied as: $\mathrm{T} 3>\mathrm{T} 4>\mathrm{T} 5>\mathrm{T} 7>\mathrm{T} 2>\mathrm{T} 6>\mathrm{T} 1$. Differences between $\mathrm{T} 3, \mathrm{~T} 4$ and $\mathrm{T} 2$ were rather marginal and did not differ significantly. Almost similar pattern was recorded during 2015-16 (Table 6).

Table.3 Tillers hill ${ }^{-1}$ in rice genotype HUR 105 at different stages of growth and treatments (2014-15)

\begin{tabular}{|c|c|c|c|c|c|c|c|}
\hline \multirow[t]{2}{*}{ S.No. } & \multirow[t]{2}{*}{ \# Treatment } & \multicolumn{6}{|c|}{ Tiller number atdifferent days after transplanting } \\
\hline & & 20 & 40 & 60 & 80 & Maturity & Mean \\
\hline 1 & T1 & 2.67 & 7.33 & 8.00 & 8.33 & 9.00 & 7.07 \\
\hline 2 & T2 & 4.33 & 10.00 & 11.67 & 11.67 & 12.00 & 9.93 \\
\hline 3 & T3 & 6.00 & 12.00 & 16.67 & 16.00 & 13.67 & 12.87 \\
\hline 4 & T4 & 4.67 & 11.00 & 16.00 & 15.33 & 13.33 & 12.07 \\
\hline 5 & T5 & 4.67 & 8.00 & 12.33 & 12.00 & 11.00 & 9.60 \\
\hline 6 & T6 & 4.00 & 9.67 & 12.33 & 11.00 & 11.33 & 9.67 \\
\hline 7 & T7 & 4.67 & 8.00 & 12.33 & 11.67 & 11.67 & 9.67 \\
\hline \multicolumn{2}{|r|}{ Mean } & 4.43 & 9.43 & 12.76 & 12.29 & 11.71 & \\
\hline \multicolumn{2}{|c|}{ Particulars } & \multicolumn{3}{|c|}{ SEm \pm} & \multicolumn{3}{|c|}{$\mathrm{CD}(\mathrm{P} \leq \mathbf{0 . 0 5})$} \\
\hline \multicolumn{2}{|c|}{ Treatment } & \multicolumn{3}{|c|}{0.28} & \multicolumn{3}{|c|}{0.81} \\
\hline \multicolumn{2}{|c|}{ Stage } & \multicolumn{3}{|c|}{0.24} & \multicolumn{3}{|c|}{0.68} \\
\hline \multicolumn{2}{|c|}{ Treatment $\times$ Stage } & \multicolumn{3}{|c|}{0.64} & \multicolumn{3}{|c|}{1.80} \\
\hline \multicolumn{8}{|c|}{$\begin{array}{l}\text { \# (Control; NPK 00:00:00)T1, (recommended dose of fertilizer: RDF; NPK 150:60:60) T2, (RDF + S:Zn:B 40:05:1.5) T3, } \\
\text { (Customized fertilizer of Tata Chemical N:P:K:Zn:B: 11:32:13:0.9:0.24\% @ } 375 \mathrm{~kg} \mathrm{ha}^{-1}+179.34 \mathrm{~kg} \mathrm{ha}{ }^{-1} \text { urea in splits) T4, 75\% } \\
\text { RDF + 25\% N through sewage sludge (T5), 75\% RDF + 25\% N through vermicompost (T6), 75\% RDF + 25\% N through sesbania } \\
\text { (GM) (T7). } \\
\text { * Statistically not significant }\end{array}$} \\
\hline
\end{tabular}


Table.4 Tillers hill ${ }^{-1}$ in rice genotype HUR 105 at different stages of growth and treatments (2015-16)

\begin{tabular}{|c|c|c|c|c|c|c|c|}
\hline \multirow[t]{2}{*}{ S.No. } & \multirow[t]{2}{*}{ \# Treatment } & \multicolumn{6}{|c|}{ Tiller number at different days after transplanting } \\
\hline & & 20 & 40 & 60 & 80 & Maturity & Mean \\
\hline 1 & T1 & 5.33 & 6.33 & 7.67 & 7.33 & 8.00 & 6.93 \\
\hline 2 & T2 & 6.67 & 11.67 & 12.00 & 12.00 & 11.33 & 10.73 \\
\hline 3 & T3 & 9.67 & 14.00 & 16.33 & 16.33 & 16.00 & 14.47 \\
\hline 4 & T4 & 9.33 & 13.67 & 16.33 & 16.00 & 15.33 & 14.13 \\
\hline 5 & T5 & 7.67 & 11.33 & 12.33 & 11.67 & 12.67 & 11.13 \\
\hline 6 & T6 & 7.33 & 11.67 & 12.67 & 12.00 & 12.00 & 11.13 \\
\hline 7 & T7 & 7.33 & 11.33 & 11.33 & 12.33 & 11.33 & 10.73 \\
\hline \multicolumn{2}{|r|}{ Mean } & 7.62 & 11.43 & 12.67 & 12.52 & 12.38 & \\
\hline \multicolumn{2}{|c|}{ Particulars } & \multicolumn{3}{|c|}{ SEm \pm} & \multicolumn{3}{|c|}{$C D(P \leq 0.05)$} \\
\hline \multicolumn{2}{|c|}{ Treatment } & \multicolumn{3}{|c|}{0.19} & \multicolumn{3}{|c|}{0.55} \\
\hline \multicolumn{2}{|c|}{ Stage } & \multicolumn{3}{|c|}{0.16} & \multicolumn{3}{|c|}{0.47} \\
\hline \multicolumn{2}{|c|}{ Treatment $\times$ Stage } & \multicolumn{3}{|c|}{0.44} & \multicolumn{3}{|c|}{1.23} \\
\hline
\end{tabular}

\section{Leaf area $\left(\mathrm{cm}^{2}\right.$ hill $\left.^{-1}\right)$}

Leaf area $\left(\mathrm{cm}^{2}\right.$ hill $\left.^{-1}\right)$ was determine at 20, 40, 60 and 80 DAT. Differences were significance with respect to treatment, stage and treatment $\times$ stage (Table 7). Leaf area hill ${ }^{1}$ under all treatments, increased progressively and attained the maximum at $60 \mathrm{DAT}$, then declined. As compared to control (T1) significantly highest leaf area hill $^{-1}$ was recorded even at 20 DAT in plants under different treatments. At this stage (20 DAT) the maximum $\left(113.77 \mathrm{~cm}^{2}\right.$ hill'-1) leaf area was recorded in plants under (T3) treatment and the minimum $\left(39.00 \mathrm{~cm}^{2}\right.$ hill-1) in plants under $\mathrm{T} 1$ treatment.

At 80 DAT this difference persisted and leaf area under different treatment varied as: T3 $\left(925.53 \mathrm{~cm}^{2} \mathrm{hill}^{-1}\right)>\mathrm{T} 4>\mathrm{T} 5>\mathrm{T} 2>\mathrm{T} 6>\mathrm{T} 7$ $>\mathrm{T} 1\left(255.57 \mathrm{~cm}^{2}\right.$ hill $\left.^{-1}\right)$.Similar pattern was observed during 2015-16 (Table 8).During both experimental years though the maximum leaf area hill ${ }^{-1}$ was recorded in plant under T3 treatment but the differences between T3, T4 and $\mathrm{T} 2$ treatments were marginal.

\section{Total plant dry weight $\left(\mathrm{g}\right.$ hill $\left.^{-1}\right)$}

Total plant dry weight $\left(\mathrm{g} \mathrm{hill}^{-1}\right.$ ) was recorded during 2014-15 and presented in Table 9). Differences were significant with respect to treatment, stage and treatment $\times$ stage. On an average total dry weight hill $^{-1}$ increased steadily from transplanting to maturity. Significant increase in total dry matter was recorded between 60 DAT to the maturity stage. At maturity stage the maximum dry weight $\left(47.69\right.$ hill $\left.^{-1}\right)$ was recorded in plants were transplanted under the $\mathrm{T} 3$ treatment and minimum (24.85 hill $\left.{ }^{-1}\right)$ in plants under $\mathrm{T} 1$ treatment. Mean total plant dry weight hill ${ }^{-1}$ under different treatments varied as: $\mathrm{T} 3>\mathrm{T} 4$ $>\mathrm{T} 7>\mathrm{T} 2>\mathrm{T} 5>\mathrm{T} 6>\mathrm{T} 1$.

Almost similar trends were recorded during 2015-16 (Table 10). As compared to the 2014-15, during 2015-16 under all treatments total plant dry weight hill $^{-1}$ was higher. During the both experimentation years differences between T3 and T4 were marginal. 
Table.5 Leaf number hill ${ }^{-1}$ in rice genotype HUR 105 at different stages of growth and treatments (2014-15)

\begin{tabular}{|c|c|c|c|c|c|c|}
\hline \multirow[t]{2}{*}{ S.No. } & \multirow[t]{2}{*}{ \#Treatment } & \multicolumn{5}{|c|}{ Leaf number at different days after transplanting } \\
\hline & & 20 & 40 & 60 & 80 & Mean \\
\hline 1 & T1 & 7.00 & 19.00 & 33.33 & 22.00 & 20.33 \\
\hline 2 & $\mathbf{T} 2$ & 12.00 & 31.00 & 47.33 & 39.33 & 32.42 \\
\hline 3 & T3 & 15.67 & 37.33 & 62.00 & 51.00 & 41.50 \\
\hline 4 & T4 & 13.33 & 33.33 & 60.33 & 48.00 & 38.75 \\
\hline 5 & T5 & 11.33 & 28.00 & 50.00 & 41.00 & 32.58 \\
\hline 6 & T6 & 11.00 & 30.33 & 47.33 & 37.67 & 31.58 \\
\hline 7 & $\mathbf{T 7}$ & 12.00 & 27.67 & 47.67 & 39.67 & 31.75 \\
\hline \multicolumn{2}{|c|}{ Mean } & 11.76 & 29.52 & 49.71 & 39.81 & \\
\hline \multicolumn{2}{|c|}{ Particulars } & \multicolumn{3}{|c|}{ SEm \pm} & \multicolumn{2}{|c|}{$C D(P \leq 0.05)$} \\
\hline \multicolumn{2}{|c|}{ Treatment } & \multicolumn{3}{|c|}{1.04} & \multicolumn{2}{|c|}{2.96} \\
\hline \multicolumn{2}{|c|}{ Stage } & \multicolumn{2}{|c|}{0.79} & & \multicolumn{2}{|c|}{2.24} \\
\hline \multicolumn{2}{|c|}{ Treatment $\times$ Stage } & \multicolumn{2}{|c|}{2.09} & & \multicolumn{2}{|c|}{5.93} \\
\hline
\end{tabular}

\# (Control; NPK 00:00:00)T1, (recommended dose of fertilizer: RDF; NPK 150:60:60) T2, (RDF + S:Zn:B 40:05:1.5) T3, (Customized fertilizer of Tata Chemical N:P:K:Zn:B: 11:32:13:0.9:0.24\% @ $375 \mathrm{~kg} \mathrm{ha}^{-1}+179.34 \mathrm{~kg} \mathrm{ha}^{-1}$ urea in splits) T4, 75\% $\mathrm{RDF}+25 \% \mathrm{~N}$ through sewage sludge (T5), $75 \% \mathrm{RDF}+25 \% \mathrm{~N}$ through vermicompost (T6), $75 \% \mathrm{RDF}+25 \% \mathrm{~N}$ through sesbania (GM) (T7). * Statistically not significant.

Table.6 Leaf number hill ${ }^{-1}$ in rice genotype HUR 105 at different stages of growth and treatments (2015-16)

\begin{tabular}{|c|c|c|c|c|c|c|}
\hline \multirow[t]{2}{*}{ S.No. } & \multirow[t]{2}{*}{ \#Treatment } & \multicolumn{5}{|c|}{ Leaf number at different days after transplanting } \\
\hline & & 20 & 40 & 60 & 80 & Mean \\
\hline 1 & T1 & 14.67 & 19.00 & 32.67 & 24.00 & 22.58 \\
\hline 2 & $\mathbf{T} 2$ & 18.67 & 39.00 & 53.00 & 42.33 & 38.25 \\
\hline 3 & T3 & 29.00 & 46.00 & 61.00 & 51.00 & 46.75 \\
\hline 4 & T4 & 27.67 & 43.00 & 60.67 & 49.67 & 45.25 \\
\hline 5 & T5 & 22.33 & 37.67 & 49.67 & 39.33 & 37.25 \\
\hline 6 & T6 & 21.00 & 38.00 & 49.00 & 41.00 & 37.25 \\
\hline 7 & $\mathbf{T 7}$ & 21.33 & 37.33 & 44.67 & 41.00 & 36.08 \\
\hline \multicolumn{2}{|c|}{ Mean } & 22.10 & 37.14 & 50.10 & \multirow{2}{*}{\multicolumn{2}{|c|}{$\begin{array}{c}41.19 \\
C D(P \leq 0.05)\end{array}$}} \\
\hline \multicolumn{2}{|c|}{ Particulars } & \multicolumn{3}{|c|}{ SEm \pm} & & \\
\hline \multicolumn{2}{|c|}{ Treatment } & \multicolumn{3}{|c|}{0.81} & \multicolumn{2}{|c|}{2.30} \\
\hline \multicolumn{2}{|c|}{ Stage } & \multicolumn{2}{|c|}{0.61} & & \multicolumn{2}{|c|}{1.74} \\
\hline \multicolumn{2}{|c|}{ Treatment $\times$ Stage } & \multicolumn{2}{|c|}{1.62} & & \multicolumn{2}{|c|}{4.61} \\
\hline
\end{tabular}

\# (Control; NPK 00:00:00)T1, (recommended dose of fertilizer: RDF; NPK 150:60:60) T2, (RDF + S:Zn:B 40:05:1.5) T3, (Customized fertilizer of Tata Chemical N:P:K:Zn:B: 11:32:13:0.9:0.24\% @ $375 \mathrm{~kg} \mathrm{ha}^{-1}+179.34 \mathrm{~kg} \mathrm{ha}^{-1}$ urea in splits) T4, 75\% $\mathrm{RDF}+25 \% \mathrm{~N}$ through sewage sludge (T5), $75 \% \mathrm{RDF}+25 \% \mathrm{~N}$ through vermicompost (T6), $75 \% \mathrm{RDF}+25 \% \mathrm{~N}$ through sesbania (GM) (T7).

* Statistically not significant 
Table.7 Leaf area $\left(\mathrm{cm}^{2}\right.$ hill $\left.{ }^{-1}\right)$ in rice genotype HUR 105 at different stages of growth and treatments $(2014-15)$

\begin{tabular}{|c|c|c|c|c|c|c|}
\hline \multirow[t]{2}{*}{ S.No. } & \multirow[t]{2}{*}{ \#Treatment } & \multicolumn{5}{|c|}{ Leaf area at different days after transplanting } \\
\hline & & 20 & 40 & 60 & 80 & Mean \\
\hline 1 & T1 & 39.00 & 159.56 & 451.29 & 255.57 & 226.36 \\
\hline 2 & $\mathbf{T} 2$ & 87.32 & 313.14 & 855.70 & 754.43 & 502.65 \\
\hline 3 & T3 & 113.77 & 464.61 & 991.61 & 925.53 & 623.88 \\
\hline 4 & T4 & 101.19 & 346.13 & 969.42 & 860.27 & 569.25 \\
\hline 5 & T5 & 96.83 & 258.16 & 841.16 & 761.77 & 489.48 \\
\hline 6 & T6 & 87.20 & 250.34 & 809.00 & 731.93 & 469.62 \\
\hline 7 & T7 & 95.43 & 246.42 & 833.56 & 716.40 & 472.95 \\
\hline \multicolumn{2}{|c|}{ Mean } & 88.68 & 291.19 & 821.68 & \multirow{2}{*}{\multicolumn{2}{|c|}{$\begin{array}{c}715.13 \\
C D(P \leq 0.05)\end{array}$}} \\
\hline \multicolumn{2}{|c|}{ Particulars } & \multicolumn{3}{|c|}{ SEm \pm} & & \\
\hline \multicolumn{2}{|c|}{ Treatment } & \multicolumn{3}{|c|}{9.31} & \multicolumn{2}{|c|}{26.46} \\
\hline \multicolumn{2}{|c|}{ Stage } & \multicolumn{2}{|c|}{7.04} & & \multicolumn{2}{|c|}{19.99} \\
\hline \multicolumn{2}{|c|}{ Treatment $\times$ Stage } & \multicolumn{2}{|c|}{18.61} & & \multicolumn{2}{|c|}{52.91} \\
\hline
\end{tabular}

\# (Control; NPK 00:00:00)T1, (recommended dose of fertilizer: RDF; NPK 150:60:60) T2, (RDF + S:Zn:B 40:05:1.5) T3, (Customized fertilizer of Tata Chemical N:P:K:Zn:B: 11:32:13:0.9:0.24 \% @ $375 \mathrm{~kg} \mathrm{ha}^{-1}+179.34 \mathrm{~kg} \mathrm{ha}^{-1}$ urea in splits) T4, 75\% $\mathrm{RDF}+25 \% \mathrm{~N}$ through sewage sludge (T5), $75 \% \mathrm{RDF}+25 \% \mathrm{~N}$ through vermicompost (T6), $75 \% \mathrm{RDF}+25 \% \mathrm{~N}$ through sesbania (GM) (T7).* Statistically not significant.

Table.8 Leaf area $\left(\mathrm{cm}^{2}\right.$ hill $\left.{ }^{-1}\right)$ in rice genotype HUR 105 at different stages of growth and treatments (2015-16)

\begin{tabular}{|c|c|c|c|c|c|c|}
\hline \multirow[t]{2}{*}{ S .No. } & \multirow[t]{2}{*}{ \#Treatment } & \multicolumn{5}{|c|}{ Leaf area at different days after transplanting } \\
\hline & & 20 & 40 & 60 & 80 & Mean \\
\hline 1 & T1 & 54.70 & 160.94 & 453.93 & 301.16 & 242.68 \\
\hline 2 & $\mathbf{T 2}$ & 114.17 & 350.52 & 847.92 & 823.84 & 534.11 \\
\hline 3 & T3 & 185.73 & 523.93 & 1082.77 & 983.71 & 694.04 \\
\hline 4 & T4 & 182.67 & 472.41 & 1019.60 & 929.47 & 651.04 \\
\hline 5 & T5 & 109.87 & 274.89 & 843.57 & 728.85 & 489.30 \\
\hline 6 & T6 & 109.40 & 273.07 & 820.70 & 775.81 & 494.74 \\
\hline 7 & $\mathbf{T 7}$ & 110.80 & 267.55 & 742.13 & 779.74 & 475.06 \\
\hline \multicolumn{2}{|c|}{ Mean } & 123.90 & 331.90 & 830.09 & 760.37 & \\
\hline \multicolumn{2}{|c|}{ Particulars } & \multicolumn{2}{|c|}{ SEm \pm} & \multicolumn{3}{|c|}{$\mathrm{CD}(\mathrm{P} \leq \mathbf{0 . 0 5})$} \\
\hline \multicolumn{2}{|c|}{ Treatment } & \multicolumn{2}{|c|}{14.15} & \multicolumn{3}{|c|}{40.24} \\
\hline \multicolumn{2}{|c|}{ Stage } & \multicolumn{2}{|c|}{10.70} & \multicolumn{3}{|c|}{30.41} \\
\hline \multicolumn{2}{|c|}{ Treatment $\times$ Stage } & \multicolumn{2}{|c|}{28.31} & \multicolumn{3}{|c|}{80.48} \\
\hline
\end{tabular}

\# (Control; NPK 00:00:00)T1, (recommended dose of fertilizer: RDF; NPK 150:60:60) T2, (RDF + S:Zn:B 40:05:1.5) T3, (Customized fertilizer of Tata Chemical N:P:K:Zn:B: 11:32:13:0.9:0.24 \% @ $375 \mathrm{~kg} \mathrm{ha}^{-1}+179.34 \mathrm{~kg} \mathrm{ha}^{-1}$ urea in splits) T4, 75\% $\mathrm{RDF}+25 \% \mathrm{~N}$ through sewage sludge (T5), $75 \% \mathrm{RDF}+25 \% \mathrm{~N}$ through vermicompost (T6), $75 \% \mathrm{RDF}+25 \% \mathrm{~N}$ through sesbania (GM) (T7).

* Statistically not significant 
Table.9 Total dry weight $\left(\mathrm{g}\right.$ hill $\left.{ }^{-1}\right)$ in rice genotype HUR 105 at different stages of growth and treatments (2014-15)

\begin{tabular}{|c|c|c|c|c|c|c|c|}
\hline \multirow[t]{2}{*}{ S.No. } & \multirow[t]{2}{*}{ \# Treatment } & \multicolumn{6}{|c|}{ Total dry weight at different days after transplanting } \\
\hline & & 20 & 40 & 60 & 80 & Maturity & Mean \\
\hline 1 & T1 & 0.50 & 1.67 & 5.64 & 6.58 & 24.85 & 7.85 \\
\hline 2 & $\mathbf{T} 2$ & 0.66 & 2.63 & 12.41 & 14.76 & 38.15 & 13.72 \\
\hline 3 & T3 & 0.80 & 3.81 & 16.39 & 18.75 & 47.69 & 17.49 \\
\hline 4 & $\mathbf{T 4}$ & 0.75 & 3.48 & 16.04 & 17.86 & 46.25 & 16.88 \\
\hline 5 & T5 & 0.68 & 2.40 & 11.88 & 15.89 & 37.80 & 13.73 \\
\hline 6 & T6 & 0.67 & 2.57 & 12.14 & 15.84 & 37.67 & 13.78 \\
\hline 7 & T7 & 0.64 & 2.56 & 12.15 & 15.47 & 40.10 & 14.18 \\
\hline \multicolumn{2}{|r|}{ Mean } & 0.67 & 2.73 & 12.38 & 15.02 & 38.93 & \\
\hline \multicolumn{2}{|c|}{ Particulars } & \multicolumn{3}{|c|}{ SEm \pm} & \multicolumn{3}{|c|}{$\mathrm{CD}(\mathrm{P} \leq 0.05)$} \\
\hline \multicolumn{2}{|c|}{ Treatment } & \multicolumn{3}{|c|}{0.32} & \multicolumn{3}{|c|}{0.90} \\
\hline \multicolumn{2}{|c|}{ Stage } & \multicolumn{3}{|c|}{0.27} & \multicolumn{3}{|c|}{0.76} \\
\hline \multicolumn{2}{|c|}{ Treatment $\times$ Stage } & \multicolumn{3}{|c|}{0.71} & \multicolumn{3}{|c|}{2.02} \\
\hline
\end{tabular}

\# (Control; NPK 00:00:00)T1, (recommended dose of fertilizer: RDF; NPK 150:60:60) T2, (RDF + S:Zn:B 40:05:1.5) T3, (Customized fertilizer of Tata Chemical N:P:K:Zn:B: 11:32:13:0.9:0.24 \% @ $375 \mathrm{~kg} \mathrm{ha}^{-1}+179.34 \mathrm{~kg} \mathrm{ha}^{-1}$ urea in splits) T4, 75\% $\mathrm{RDF}+25 \% \mathrm{~N}$ through sewage sludge (T5), $75 \% \mathrm{RDF}+25 \% \mathrm{~N}$ through vermicompost (T6), $75 \% \mathrm{RDF}+25 \% \mathrm{~N}$ through sesbania (GM) (T7).

* Statistically not significant

Table.10 Total dry weight $\left(\mathrm{g}\right.$ hill $\left.^{-1}\right)$ in rice genotype HUR 105 at different stages of growth and treatments (2015-16)

\begin{tabular}{|c|c|c|c|c|c|c|c|}
\hline \multirow[t]{2}{*}{ S.No. } & \multirow[t]{2}{*}{ \# Treatment } & \multicolumn{6}{|c|}{ Total dry weight at different days after transplanting } \\
\hline & & 20 & 40 & 60 & 80 & Maturity & Mean \\
\hline $\mathbf{1}$ & $\mathbf{T 1}$ & 0.51 & 1.63 & 5.74 & 6.27 & 23.97 & 7.62 \\
\hline 2 & $\mathbf{T} 2$ & 0.65 & 2.87 & 12.03 & 16.20 & 41.97 & 14.74 \\
\hline 3 & T3 & 0.85 & 3.75 & 15.69 & 20.15 & 51.11 & 18.31 \\
\hline 4 & T4 & 0.84 & 3.61 & 15.01 & 19.25 & 50.96 & 17.93 \\
\hline 5 & T5 & 0.72 & 2.68 & 12.66 & 16.03 & 41.51 & 14.72 \\
\hline 6 & T6 & 0.70 & 2.63 & 12.17 & 16.47 & 41.22 & 14.64 \\
\hline 7 & T7 & 0.68 & 2.61 & 11.55 & 16.10 & 39.84 & 14.16 \\
\hline \multicolumn{2}{|c|}{ Mean } & 0.71 & 2.83 & 12.12 & 15.78 & 41.51 & \\
\hline \multicolumn{2}{|c|}{ Particulars } & \multicolumn{3}{|c|}{ SEm \pm} & \multicolumn{3}{|c|}{$\mathrm{CD}(\mathrm{P} \leq \mathbf{0 . 0 5})$} \\
\hline \multicolumn{2}{|c|}{ Treatment } & \multicolumn{3}{|c|}{0.21} & \multicolumn{3}{|c|}{0.60} \\
\hline \multicolumn{2}{|r|}{ Stage } & \multicolumn{3}{|c|}{0.18} & \multicolumn{3}{|c|}{0.51} \\
\hline \multicolumn{2}{|c|}{ Treatment $\times$ Stage } & \multicolumn{3}{|c|}{0.48} & \multicolumn{3}{|c|}{1.35} \\
\hline \multicolumn{8}{|c|}{$\begin{array}{l}\text { \# (Control; NPK 00:00:00)T1, (recommended dose of fertilizer: RDF; NPK 150:60:60) T2, (RDF + S:Zn:B 40:05:1.5) T3, } \\
\text { (Customized fertilizer of Tata Chemical N:P:K:Zn:B: 11:32:13:0.9:0.24 \% @ } 375 \mathrm{~kg} \mathrm{ha}^{-1}+179.34 \mathrm{~kg} \mathrm{ha}{ }^{-1} \text { urea in splits) T4, 75\% } \\
\text { RDF + 25\% N through sewage sludge (T5), 75\% RDF + 25\% N through vermicompost (T6), 75\% RDF + 25\% N through sesbania } \\
\text { (GM) (T7). } \\
\text { * Statistically not significant }\end{array}$} \\
\hline
\end{tabular}


It is reported that partial substitution of inorganic nitrogen by green manures, vermicompost, or poultry manure improves growth of rice (Deshpande et al., 2011; Gupta et al., 2006; Vaiyapuri et al., 1998). Digested sludge, press mud and woolen carpet wastes were also found to be effective in increasing crop growth (Dash et al., 2011). Mahendra Kumar et al., (2017) and Sonam et al., (2018) reported that $\mathrm{ZnSO}_{4}$ and borax, if applied to rice@ 20 and $4 \mathrm{~kg} \mathrm{ha}^{-1}$, respectively, increase plant height, tillers hill ${ }^{-1}$ and other growth parameters significantly.

Sanjay Kumar et al., (2015) reported increased plant height, tillers hill $^{-1}$, leaf number hill ${ }^{-1}$ and leaf area hill ${ }^{-1}$ in rice plants under integrated nutrient management and the increment was more when S, Zn and B were supplemented with RDF. In this investigation such observations were further confirmed as it is observed that as compared to (control; NPK 00:00:00) T1, the height of rice plants under other treatments was higher at all the stages of studies during 2014-15 as well as 2015-16 (Table 1;2).

At maturity plant height was the maximum in treatments where crop was supplied with inorganic sources of nutrients (T2, T3 and T4) than those which received $\mathrm{RDF}+25 \% \mathrm{~N}$ through organic sources (T5, T6 and T7). Plant height was the maximum when RDF was supplemented with S:Zn:B @ 40:05:1.5 $\mathrm{kg} \mathrm{ha}{ }^{-1}$ (T3), respectively (T3) and the differences between T3, T4 (Tata Chemical $\mathrm{N}: \mathrm{P}: \mathrm{K}: \mathrm{Zn}: \mathrm{B}: \quad$ 11:32:13:0.9:0.24\%), and T2 (RDF; NPK @ 150:60:60) were not significant. Similarly plant height under T5 (75\% RDF $+25 \% \mathrm{~N}$ through sewage sludge), T6 $(75 \% \mathrm{RDF}+25 \% \mathrm{~N}$ through vermicompost), and T7 (75\% RDF $+25 \% \mathrm{~N}$ through sesbenia (GM)) treatments did not differ significantly but values were significantly higher than in T1 (RDF). Similar effects were observed on tillers hill ${ }^{-1}$ (Table 3;
4), leaf number hill $^{-1}$ (Table 5; 6), leaf area hill $^{-1}$ (Table $\left.7 ; 8\right)$, total plant dry weight hill ${ }^{-1}$ (Table 9; 10). Significantly higher growth responses of rice genotype HUR 105 to different inorganic and organic fertilizers was evident even at 20 days after transplanting (DAT) which continued till maturity.

This investigation further proves that under existing soil fertility conditions, inorganic sources of nutrients in recommended doses along with secondary nutrient $\mathrm{S}$ and micronutrients viz. $\mathrm{Zn}$ and $\mathrm{B}$ are required for achieving the maximum growth of rice in rice-wheat cropping system. It was also evident that $25 \%$ replacement of $\mathrm{N}$ of RDF by different organic sources resulted in lesser crop growth than when crop was supplied fertilizers solely through inorganic sources, however, efficacy of all organic sources used in this experiment (sewage sludge, vermicompost and sesbenia (GM)) in regulation growth in rice has been at par. In treatments $\mathrm{T} 5$ (75\% RDF $+25 \% \mathrm{~N}$ through), T6 $(75 \% \mathrm{RDF}+25 \% \mathrm{~N}$ through vermicompost), and T7 (75\% RDF $+25 \% \mathrm{~N}$ through sesbenia) growth parameters were superior than T1 (NPK @ 00:00:00), indicated that to some extent partial amount of RDF may be supplied by sewage sludge, vermicompost or sesbenia (GM).

Application of organic and inorganic sources of nutrients improved plant height, tillers hill ${ }^{-}$ 1 , leaf number hill ${ }^{-1}$, leaf area hill ${ }^{-1}$ and total plant dry weight hill ${ }^{-1}$. It is concluded that the improvements in treatments which were provided with inorganic nutrients, that too when RDF was supplemented with $\mathrm{S}, \mathrm{Zn}$ and B. Customized fertilizer of Tata Chemical (T4) was also effective in improving these parameters, but to a lesser magnitude than T3. It was evident that for optimal growth of rice in rice-wheat cropping system application of $\mathrm{S}, \mathrm{Zn}$ and B to rice @ 40:05:1.5 is essential. Replacements of $25 \%$ inorganic nitrogen by 
sewage sludge, vermicompost, and sesbania also improved studied growth parameters and in all these treatments values of studied attributes/parameters were at par, but lower than those treatments which received nutrients exclusively through inorganic sources of (T2, T3 and T4).

\section{References}

Alam, M. M., Ladha, J. K., Rahman, Z., Khan, S. R., Khan, A. H. and Buresh, R. J. (2006). Nutrient management for increased productivity of rice-wheat cropping system in Bangladesh. Field Crops Research, 96(2-3), 374-386.

Anonymous, 2013.

Anonymous, 2015.

Baskar, K. (2003). Effect of integrated use of inorganic fertilizers and FYM or green leaf manure on uptake and nutrient use efficiency of rice-rice system on an Inceptisol. Journal of the Indian Society of Soil Science, 51(1), 47-51.

Bisht, P.S., Pandey, P.C. and Lal, P. (2002). Effect of organic and inorganic sources of nutrients on rice yield and soil nutrient status in ricewheat cropping system. In : Proceeding of the second international agronomy congress on balancing food and environmental security-a continuing changes. Nov. 2630, 2002, New Delhi. pp. 53-54.

Bodruzzaman, M., Meisner, C. A., Sadat, M. A. and Hossain, M. I. (2010). Longterm effects of applied organic manures and inorganic fertilizers on yield and soil fertility in a wheat-rice cropping pattern. In Proceedings of the 19th World Congress of Soil Science (pp. 10$15)$.

Dash, D., Patro, H., Tiwari, R. C. and Shahid, M. (2011). Effect of organic and inorganic sources of $\mathrm{N}$ on growth attributes, grain and straw yield of rice (Oryza sativa). International Journal of
Pharmacy and Life Science, 2(4): 655660.

Deshpande, H.H., Devasenapathy, P. (2011). Effect of different organic sources of nutrients and green manure on growth and yield parameters of rice (Oryza sativa L.) grown under lowland condition. Crop Research, 41(1/3): 1-5.

Gomez, K. A., and Gomez, A. A. (1984).

Two factor experiments. Statistical procedures for agricultural research, 84-130.

Gupta, V., Sharma, R. S. and Vishwakarma, S. K. (2006). Long-term effect of integrated nutrient management on yield sustainability and soil fertility of rice (Oryza sativa)-wheat (Triticum aestivum) cropping system. Indian Journal of Agronomy, 51(3): 160-164.

Hobbs, P., and Morris, M. L. (1996). Meeting South Asia's future food requirements from rice-wheat cropping systems: priority issues facing researchers in the post-green revolution era.

Hossain, M. and Singh, V. P. (2000). Fertilizer use in Asian agriculture: implications for sustaining food security and the environment. Nutrient Cycling in Agroecosystems, 57(2), 155-169.

Kumar, M. M., Subbarayappa, C. T. and Ramamurthy, V. (2017). Effect of graded levels of zinc and boron on growth, yield and chemical properties of soils under paddy. International Journal of Current Microbiology \& Applied Sciences, 6(10): 1185-1196.

Kumar, S., Srivastava, J. P., Singh, A. K., Singh, S. K., Bohra, J. S., Singh, S. P. and Singh, R. P. (2015). Growth, photosynthesis, yield and yield attributes in rice (Oryza sativa L.) under inorganic and organic source of nutrients in rice-wheat cropping system, The Ecoscan, 9 (3\&4), 987-993.

Mahajan, A. and Gupta, R. D. (2009). Integrated Nutrient 
Management (INM) in a Sustainable Rice-Wheat Cropping System. Springer Science \& Business Media.

Nambiar, K. K. M., and Abrol, I. P. (1989). Long-term fertilizer experiments in India: An overview. Fertilizer News, 34(4), 11-20.

Singha, D. D. (2003). Management of crop residue in summer rice and its effect on the soil properties and crop yield. Crop Research-Hisar, 25(1), 191-193.

Sonam, B., Jatav, S. S., Singh, S. K., Patra,
A., Jatav, H. S. and Kumar, M. (2018). Evaluation of different combination of zinc, boron and sulphur application on growth and yield of hybrid rice (Oryza sativa L.). Journal of Pharmacognosy and Phytochemistry, 7(6): 2601-2605.

Vaiyapuri, V. M., Ravichandran, S. S. and Sriramachandra, S. (1998). Effect of graded levels of Sesbania with time of $\mathrm{N}$ application on growth and yield in rice-rice sequence. Annals of Agronomy Research, 19(1): 1-3.

\section{How to cite this article:}

Sanjay Kumar and Srivastava. J.P. 2020. Morphological Responses of Rice (oryza sativa 1.) to Organic and Inorganic Nutrients in Rice-Wheat Cropping System. Int.J.Curr.Microbiol.App.Sci. 9(02): 1387-1398. doi: https://doi.org/10.20546/ijcmas.2020.902.161 